\title{
Magnetic properties of Ni(II) complexes of (hydrazone)imine 1,2,3-triketones: intramolecular exchange interaction
}

Yu.N. Shvachko, ${ }^{1}$ D.V. Starichenko, ${ }^{1}$ A.V. Korolev, ${ }^{1}$ V.V. Ustinov ${ }^{1}$ D.W. Boukhvalov, ${ }^{1,2}$ V.Yu. Irkhin, ${ }^{1}$ O.G. Khudina, ${ }^{3}$ E.V. Schegolkov, ${ }^{3}$ Ya.V. Burgart, ${ }^{3}$ V.I. Saloutin, ${ }^{3}$ O.N. Chupakhin ${ }^{3}$

${ }^{1}$ Institute of Metal Physics, 18, S. Kovalevskaya str. Ekaterinburg 620041, Russia

${ }^{2}$ Radboud University of Nijmegen, NL-6525ED Nijmegen, The Netherlands

${ }^{3}$ Institute of Organic Synthesis, 22, S. Kovalevskaya str. Ekaterinburg 620041, Russia

D.Bukhvalov@science.ru.nl

We carry out comparative study of electronic structure, magnetic and resonance properties of three novel tetracyclic Ni(II) complexes based on (hydrazone)imine 1,2,3-triketones. The work presents experimental data on the static magnetic susceptibility, magnetization and EPR lineshape, width and spin contribution, density of states for $O, C$ and $N$ atoms, local spin densities on the coordination sites. The exchange interactions and effective magnetic moments are calculated by DFT methods taking into account electronic correlations.

Single Molecule Magnets (SMMs) represent a new class of zerodimensional magnetic materials consisting of almost noninteracting, identical point-like nanoscale entities. ${ }^{1,2}$ In recent years, chelate transition metal complexes have attracted considerable interest because they hold promise for the construction of polyfunctional magneto active materials. ${ }^{3,4}$ The magnetic properties of molecular crystals based on nickel complexes are of particular inter est. Bis chelate NiII complexes containing the central $\mathrm{NiN}_{4}$ fragment, in which the metal atom is in a square planar ligand environment (coordination number is 4 ), are known to be diamagnetic. ${ }^{5}$ In these complexes, the ligand environment can be formed, in particular, by different 1,2 diamines used as the starting organic compounds, which, depending on the synthesis conditions, are either involved in the complex in the initial form or oxidized to give semidiimines or diimines-2,3 The geometry of the resulting metal chelate $\mathrm{NiN}_{4}$ unit can be controlled by varying the electronic and steric characteristicsof chelate ligands is such systems, thus resulting in considerable deviations from the planar geometry, which can give rise to paramagnetism.
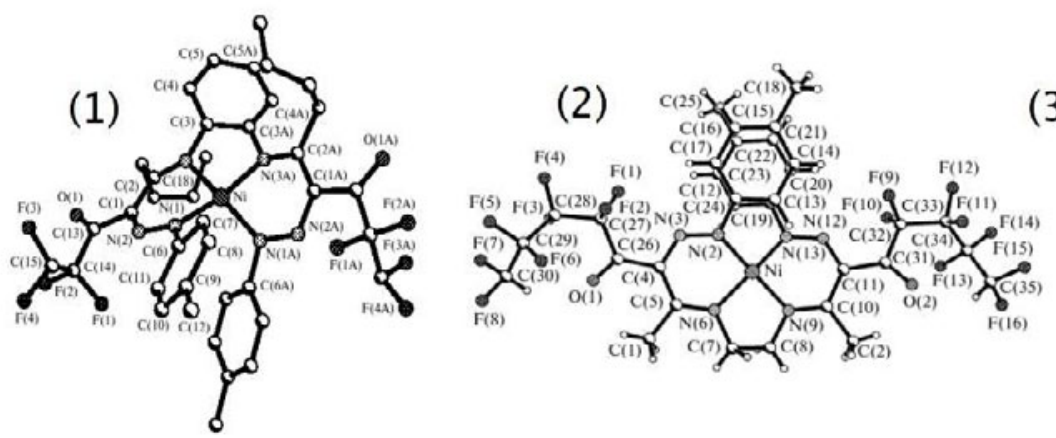

(3)

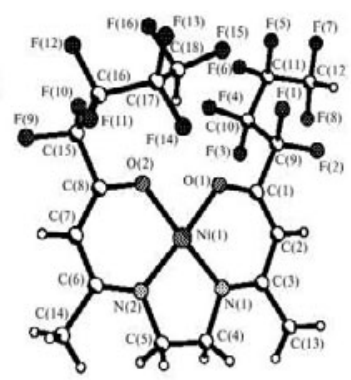

Fig.1. Molecular structure of $\mathrm{Ni}(\mathrm{II})$ complexes.

On Fig. 1 presented chemical structure of studied complexes. Details of synthesis and geometrical structure of studied complexes reported in [3]. In the complex $1 \mathrm{Ni}$ ion is in four $\mathrm{N}$ tetrahedral environment, in the complex $\mathbf{2} \mathrm{Ni}$ ion is also coordinated by four $\mathrm{N}$ but they form a plane square, in the complex $\mathbf{3} \mathrm{Ni}$ ions is in tetrahedral environment containing two $\mathrm{O}$ and two $\mathrm{N}$. Described differences in local environment of Ni ion produce a different magnetic properties 
which could be observed in magnetic measurements and by first principles calculations. The electronic properties of such Single Molecule Magnets (SMMs) can be investigated by ab initio calculations, though where the core of a SMM contains Transition Metal (TM) atoms an intrinsic difficulty is the presence of correlation effects between the $3 d$ electrons of the TM. Various SMMs have already been investigated by ab initio mehods ([6] and referencies therein).

Magnetic measurements were carried out on a SQUID MPMS5XL (QUANTUM DESIGN) magnetometer.The static magnetic susceptibility, $\chi(\mathrm{T})$, of polycrystalline samples was measured at a magnetic fields $\mathrm{B}_{0}=0.03 \div 3 \mathrm{~T}$ in the temperature range of $2 \div 293 \mathrm{~K}$. The magnetization curves $\mathrm{M}(\mathrm{B})$ were measured at $2 \mathrm{~K}$ in the field range of $0 \div 50$ $\mathrm{kG}$. The paramagnetic susceptibility was determined by subtracting the diamagnetic contributions of the organic core and the capsule, measured throughout the temperature range, from the total magnetic response. Although the geometry of the chelate site of studied complexes is similar, their magnetic properties are qualitatively different. The complex $\mathbf{3}$ is diamagnetic showing neither EPR signal nor bulk paramagnetism on SQUID, which is in agreements with the square-planar geometry of $\mathrm{NiN}_{4}$ site. In $\mathbf{1}$ and $\mathbf{3}$ the nickel atom has a strongly tetrahedrally distorted coordination environment formed by four nitrogen atoms $\mathrm{NiN}_{4}(\mathbf{1})$ or mixed nitrogen and oxygen atoms $\mathrm{NiN}_{2} \mathrm{O}_{2}(3) .{ }^{3}$ Magnetic properties of $\mathbf{1}$ are very unusual. The absolute value and temperature behavior $\chi(\mathrm{T})$ depend on the magnitude of the external magnetic field $B_{0}$ as shown in Fig. 2. At stronger fields $\mathrm{B}_{0}>1.0 \mathrm{~T} \chi(\mathrm{T})$ obeys Curie-Weiss law with $\vartheta_{\text {theor }}=-$ $200 \mathrm{~K}$. The experimental points measured at $\mathrm{B}_{0}<1.0 \mathrm{~T}$ deviates from theoretical curve $\chi(\mathrm{T})=\mathrm{C}_{\text {theor }} /\left(\mathrm{T}-\vartheta_{\text {theor }}\right)$ at $\mathrm{T}$ $<80 \mathrm{~K}$. In weaker fields $\mathrm{B}_{0}<0.5 \mathrm{~T}$ strong relaxation effect brings field and temperature irreversibility into $\chi(\mathrm{T}) \mathrm{de}-$ pendence as shown in Fig. 2. The irreversible dependence at $\mathrm{B}_{0}=0.03 \mathrm{~T}$ was recorded after zero-field cooling (ZFC) down to $2 \mathrm{~K}$ and further measurement of $\chi(\mathrm{T})$ at heating up to $200 \mathrm{~K}$. A Curie-Weiss behavior with Ctheor $=8.6$ was revealed at cooling segment from $200 \mathrm{~K}$ back to $2 \mathrm{~K}$.

A knee-like anomaly is observed at $80 \mathrm{~K}$ in both heating and cooling regimes. The character of the temperature behavior in the ranges $2 \div 80 \mathrm{~K}$ and $100 \div 200 \mathrm{~K}$ is similar. The passage through zero field leads to hesteretic behavior of $\chi(\mathrm{T})$ similar to that for anisotropic AFM systems. An ESR signal from a polycrystalline sample is characteristic of $\mathrm{Ni}$ (II) with $\triangle \mathrm{B}=$ $400 \mathrm{G}$ and the anisotropic $\mathrm{g}$ factor with $\mathrm{g}_{1}=2.203$ and $\mathrm{g}_{2}=3.378$. The normalized spectra for 
three different polycrystalline samples are presented in Fig. 3. However, further cooling down to $100 \mathrm{~K}$ is accompanied by a narrowing of the ESR signal to $170 \mathrm{G}$. The temperature evolution of the integrated intensity of the ESR signal in the range of $100 \div 350 \mathrm{~K}$ does not obey Curie's law, which is consistent with the results of SQUID measurements. A weak paramagnetism is evidenced by the small magnetic moment $\left(\sim 10^{-2} \mu_{B}\right)$ at $2 \mathrm{~K}$. It should be noted that the ESR spectrum reveals also two weak lines with $\mathrm{g}=3.164$, and 3.681. Substitution of two nitrogen atoma to oxygen in the coordination site with similar character of distortions dramatically changes the hybridization effects and respectively the EPR properties. ${ }^{7,8}$

First principles calculation was carried out for all studied complexes. Technical details of calculations is the same as in [6]. For our calculations we choice value of Coulomb repulsion is equal $4 \mathrm{eV}$ that is the same for other SMMs. ${ }^{6} \mathrm{In}$ according with different local environment of $\mathrm{Ni}$ ions and results of magnetic measurements electronic structure of all complexes is completely different (see Fig. 4). Complex $\mathbf{2}$ is non magnetic, complex $\mathbf{3}$ is typical paramagnetic with magnetic moment $1.9 \mu_{B}$ on $\mathrm{Ni}$ and neglected magnetic polarization of ligands. But electronic structure of complex 1 is different. Magnetic moment on Ni ion is about $1.6 \mu_{B}$ but magnetic polarization of ligands is not neglected. Calculated values of magnetic moments on nitrogens, oxygens and carbons is about $0.05 \mu_{B}$ and total magnetic moment on ligand is $0.4 \mu_{B}$ and one oriented antiparallel to magnetic moment on Ni ions. Described results is unusual for SMMs but all well studied SMMs contain no similar rings with $\pi$ bonds. Well studied experimentally and theoretically ${ }^{9}$ carbon based systems which contain honeycomb lattices could be a good model for understanding the nature of magnetism in studied systems. They contain a similar rings with $\pi$ -

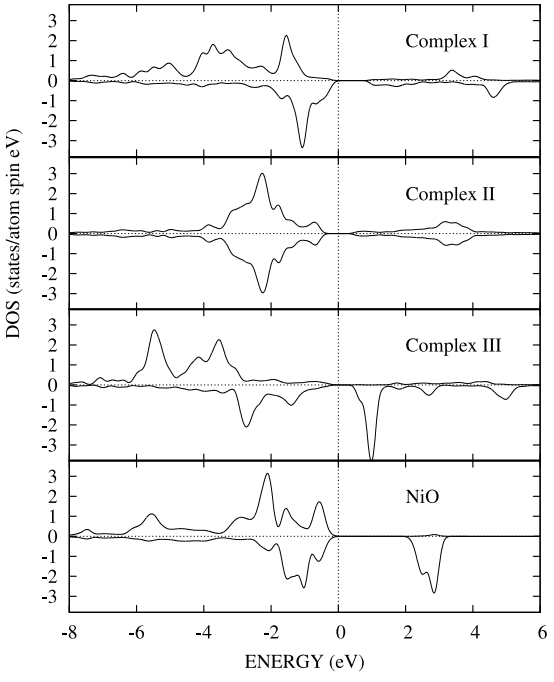

Fig.4. Densities of states for $3 d$ orbitals of $\mathrm{Ni}$ complexes and $\mathrm{NiO}$.

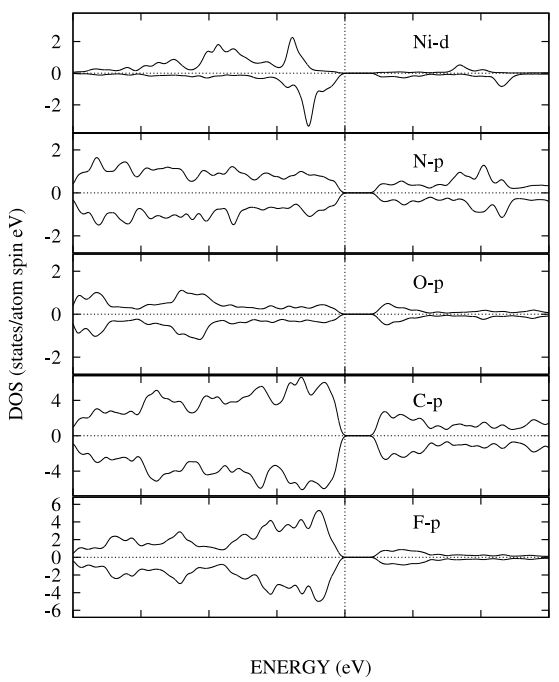

Fig.5. Partial densities of states for $\mathrm{Ni}$ ion and ligands of complex $\mathbf{1}$. bonds.

On Fig. 5 presented partial densities of states for light elements from ligands in complex 1. We can see that $p$ bands in this complex is more wide than in standard $\mathrm{SMMs}^{6}$ and similar to $p$ bands of carbon in fullerenes. ${ }^{9}$ Since first report about ferromagnetism in polymerized fullerenes[10] they attract much attention. In resent theoretical works was described the vacancies ${ }^{11}$ or chemical doping ${ }^{12}$ of $\mathrm{C}_{60}$ as a case of ferromagnetism. Received results qualitatively correspond with experimentally observed magnetism on carbon in proton irradiated graphite ${ }^{13}$ and $\mathrm{Fe} / \mathrm{C}$ multilayers. ${ }^{14}$ In [12] was detailed studying distribution on magnetisation on fullerene cage and shown that magnetic moments distributed on shell equivalently. This result correspond with experimentally observed magnetism in systems where far laying magnetic centres separated by fullerenes. ${ }^{15,16}$ Therefore magnetisation of light elements in systems which 
contain $\pi$-bonds could be a case of long range magnetic interaction. Value and sign of exchange interaction depend from chemical and geometrical structure of material. Antiparallel orientation of magnetic moments on metal ions and ligands can be describe in terms of coordinate bonds. As against strong covalent bond where magnetic polarisation is the same of all atoms in weak coordinate bonds antparallel orientation of magnetic moments on metal and ligands intensify bonds. In standard situations where magnetic ions coordinated by non interactive molecules or light elements without free $\pi$-electrons (as in complex 3) ligands have no collectivized electrons for provide not neglected magnetic moment in wide area but for ligands of complex $\mathbf{1}$ and carbon systems with honeycomb lattices it possible. For estimate the value of $\mathrm{T}_{N}$ for complex 1 we performed calculation of total energies for supersell contain two monomer with parallel and antiparallel orientation of magnetic moments on $\mathrm{Ni}$ ions. We find that total energy of antiferromagnetic configuration is lower ant estimated from energy difference value of $\mathrm{T}_{N}$ is $54 \mathrm{~K}$ which is correspond with experimental results.

The comparative analysis of magnetic, resonance and electronic properties of the set functionally related $\mathrm{Ni}$ (II) (hydrazone)imine 1,2,3-triketones indicate the involvement of nitrogen p-orbitals of coordination site to the hybridization processes. The tetrahedral distortions have an effect on the spin state of $\mathrm{Ni}(\mathrm{II})$. It is concluded that physical properties of studied complexes are sensitive to chemical composition and entire symmetry of the molecule. The model suggested takes into account the intramolecular exchange induced by the hybridization of $\mathrm{Ni}$ (II) $d$-states and $s p$-orbitals of the atoms of the chelate site. The spin polarization on the atoms of the chelate site is experimentally found. The existence of two spin subsystems localized on $d$ and $s p$-states leads to the antiferromagnetic behavior of the effective magnetic moment.

This study was financially supported by the Russian Science Support Foundation, the Council on Grants of the President of the Russian Federation (Program for State Support of Leading Scientific Schools of the Russian Federation, Grants NSH - 9178.2006.3, NSH -4640.2006.2 and NSH - 5869.2006.2), the Russian Science Foundation for Basic Research (Project No. 0502-17704), the Siberian Branch of the Russian Academy of Sciences (Program No. 29), and the Ural Branch of the Russian Academy of Sciences (Program No. 24). D. W. Boukhvalov acknowledges support from Stichting voor Fundamenteel Onderzoek der Materie (FOM), the Netherlands.

1. O. Kahn, Molecular Magnetism VCH, New York (1993).

2. D. Gatteschi, A. Caneschi, L. Pardi, and R. Sessoli, Science 2651054 (1994).

3. O.G. Khudina et al., Russian Chem. Bull., Int.Ed. 56, 103 (2007).

4. V. I. Ovcharenko and R. Z. Sagdeev, Russ. Chem. Revs. 68, 381 (1999).

5. T. Ishizuki, S. M. Peng, Y. Wang, and Ch. K. Chiang, Acta Chystallogr., Sect. C 40, 1541 (1984).

6. D. W. Boukhvalov et al., Phys. Rev. B 75, 014419 (2007).

7. R. L. Carlin, Magnetochemistry Springer, Berlin (1986).

8. A. F. Orchar, Magnetochemistry Oxford University Press, Oxford (2003).

9. D. W. Boukhvalov et al., Phys. Rev. B 69, 115425 (2004).

10. T.L. Makarova et al., Nature (London) 413, 716 (2001).

11. D. W. Boukhvalov and M.I. Katsnelson (to be published).

12. O. E. Kvyatkovskii et al., Phys. Rev. B 72, 214426 (2005).

13. H. Ohldag et al., Phys. Rev. Lett. 98, 187204 (2007).

14. H.-Ch. Mertings et al., Europhys. Lett. 66, 743 (2004).

15. P.M. Allemand et al., Science 253, 301 (1991).

16. D.V. Konarev et al., J. Am. Chem. Soc. 128, 9292 (2006). 